\title{
Die Marknagelung des Femurs: eine Standortbestimmung
}

\author{
$\square$ D. Höntzsch, S. Weller, K. Weise
}

\section{Zusammenfassung}

Der große Vorteil der Marknagelung ist, dass der vom Osteosynthesematerial übernommene Kraftfluss in der natürlichen Achse des Knochens liegt. Es ist somit die Osteosynthese, welche den physiologischen Umständen am nächsten kommt. Als Wegbereiter ist Gerhard Küntscher zu nennen, welcher 1939 die erste Marknagelung einer Oberschenkelschaftfraktur durchführte. In den Anfangsjahren wurde die Marknagelung in unaufgebohrter Technik durchgeführt. Somit wurde mit ihr keine neue Osteosynthese eingeführt, sondern mit besserer Technik und besseren Materialien kehrt die Marknagelung zu ihren Wurzeln zurück. Durch die Marknagelung kommt es dank physiologischer Fragmentbewegungen zu einer gewollten und positiv zu beurteilenden indirekten Frakturheilung. Die Marknagelung kann als minimal-invasive Osteosynthesemethode bezeichnet werden. Durch die Verriegelung und die unaufgebohrte Marknageltechnik konnte das Indikationsspektrum gegenüber der klassischen Marknagelung wesentlich erweitert werden. Heute ist es möglich, alle Frakturen, welche zwischen den Verriegelungslöchern liegen, mit der Marknagelung zu versorgen. Die proximale Verriegelung ist einfach über Bügelsysteme möglich, die distale Verriegelung erfordert spezielle Techniken. Unaufgebohrte und aufgebohrte Marknagelungen dürfen nicht als Gegensatz gesehen werden, beide haben ihre Vor- und Nachteile sowie Regeln, die beachtet werden müssen. Beim Beachten dieser Regeln sollte es gelingen, die Aspekte der Biologie und Stabilität mit aufgebohrten und unaufgebohrten Marknagelungen in Balance zu bringen. Die Marknagelung erfordert eine sorgfältige Lagerung, welche vom Operateur oder einem qualifizierten Vertreter zusammen mit dem gesamten Operationsteam durchgeführt werden muss. Die Übersicht der Marknagelung zeigt, dass heute alle langen Röhrenknochen Femur, Tibia, Humerus und der Unterarm bis in Gelenknähe erfolgreich mit den verschiedenen Techniken stabilisiert werden können.

schen Chirurgenkongress 1940 berichtete er über seine ersten Fälle.

In den 40er Jahren gab es erste Ansätze, Kirschner-Drähte und Pins auch zum Teil intramedullär einzubringen.

Als Wegbereiter ist Gerhard Küntscher (Abb.1) zu nennen, welcher 1939 die erste Marknagelung einer Oberschenkelfraktur durchführte [1]. Auf dem Deut-

OP-JOURNAL 2000; 16: 164-168

(C) Georg Thieme Verlag Stuttgart · New York
In den 50er Jahren war der Erfolg der Marknagelung aber schon so verbreitet, dass auch Böhler diese als eine der geeignetsten Osteosynthesen angesehen hat.

Wichtig zu wissen ist, dass die Marknagelung bis 1955 unaufgebohrt durchgeführt wurde. Probleme zur damaligen Zeit war,

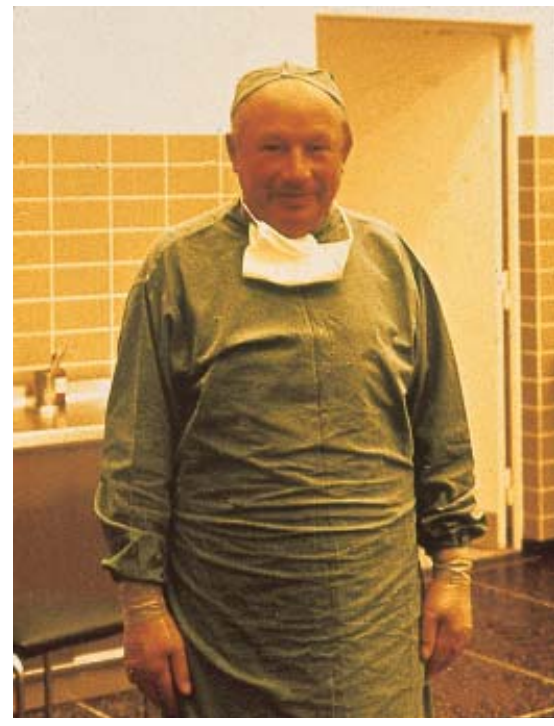

Abb.1 Gerhard Küntscher - Der Pionier der Marknagelung in seinem OP.

dass nach der Nagelung eine Instabilität verblieben ist, oder dass, wenn der Nagel zu dick gewählt wurde, es zu einem Verklemmen desselben kommen konnte. Dies führte dann ab 1954/55 zur ersten Aufbohrung mit starren Bohrern und später mit den heute noch bekannten Bohrern mit flexiblen Wellen.

In den Anfangsjahren wurde die Marknagelung in unaufgebohrter Technik durchgeführt.

Somit wurde mit der Marknagelung ohne Aufbohrung keine neue Technik eingeführt, sondern mit besserer Technik und besseren Materialien kehrt die Marknagelung zu ihren natürlichen Wurzeln zurück!

In den folgenden Jahren wurden verschiedene Nagelprofile ausprobiert. Alle großen Hersteller endeten mit ihrem Design bei geschlitzten Rohrnägeln mit nur leicht differierendem Querschnittdesign [2]. 
Bereits Küntscher und seine Schüler Maatz und Herzog haben wesentliche Schritte der Weiterentwicklung beschrieben [1]:

- Gammanagel als Vorläufer aller proximalen Femurnägel,

- Detensornagel als Vorläufer der Verriegelungsmarknagelung u. a.

Beginnend mit den 70er und 80er Jahren kam es dann zu einer raschen Fortentwicklung der Verriegelungsmarknagelung in unaufgebohrter und aufgebohrter Technik bis zum heutigen Status.

\section{Biomechanik und Frakturheilung [2]}

Zur Marknagelung werden alle im Markkanal (intramedullär) gelegenen Kraftträger gerechnet. Der große Vorteil der Marknagelung ist, dass der vom Osteosynthesematerial übernommene Kraftfluss in der natürlichen Achse des verletzten Knochens (nämlich im Zentrum) liegt. Es ist somit die Osteosynthese, welche den physiologischen Umständen am nächsten kommt. Hierdurch wird mit geringstmöglichem Material die höchstmögliche Stabilität für eine funktionelle Nachbehandlung und zumindest Teilund in vielen Fällen rasche Vollbelastung erreicht.

\section{Frakturheilung}

Nur ausnahmsweise kann und wird mit der Marknagelung auch eine interfragmentäre Kompression mit Kompressionsnägeln erreicht. Die Regel ist, dass physiologische Bewegungen im Frakturbereich möglich sind. Dadurch und durch das minimal-invasive Vorgehen mit im Regelfall „No-touch“-Technik (geschlossenes Verfahren) findet eine sekundäre, d.h. indirekte Frakturheilung mit mehr oder wenig großer spindelförmiger Kallusbildung statt (Abb.2 u. 3).

Wenn das Wort nicht durch eine spätere Entwicklung in der Viszeralchirurgie besetzt wäre, könnte man sagen, dass die Marknagelung die erste ,minimal-invasive Osteosynthesemethode“ gewesen ist.

Ein mehr oder weniger großes Verletzungsgebiet am Knochen und im Bereich der Weichteile wird nicht direkt angegangen, vielmehr wird weit weg vom Ort des Geschehens mit einer kleinen Inzision ein zentraler Kraftträger eingebracht. Wenn eine zusätzliche Stabilisierung notwendig ist, wird diese an den jeweiligen Enden, nämlich am Eintritts-
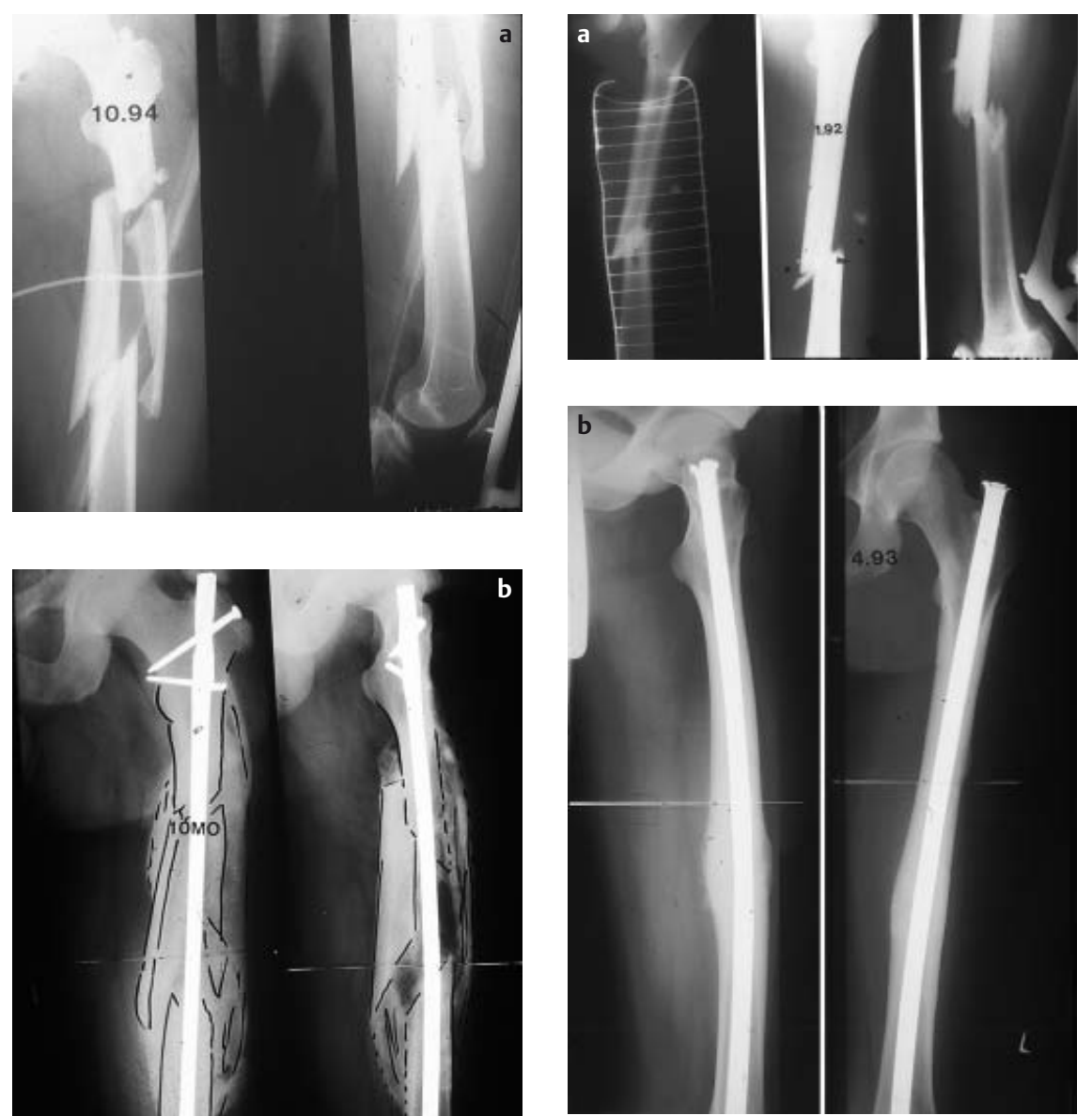

Abb. 3 Oberschenkelschaft-Querfraktur, versorgt mit aufgebohrtem, unverriegeltem Marknagel - mit spindelförmiger Kallus.

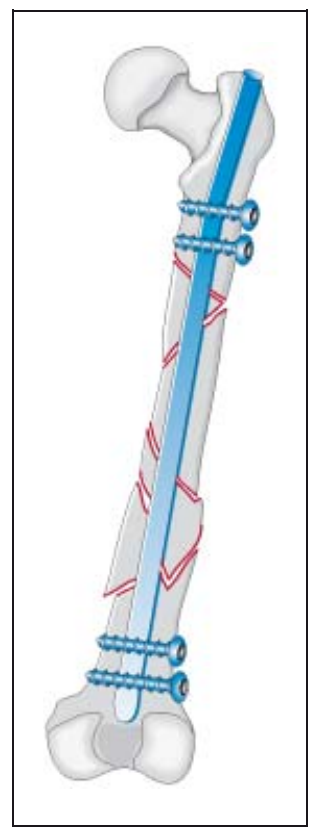

Abb. 4 Körpernahe und körperferne Verriegelung einer Oberschenkeltrümmerfraktur wie bei Abb. 2
Abb.2a-c Spindelförmige sekundäre bzw. indirekte Kallusheilung einer Oberschenkeltrümmerfraktur. 
und am Endpunkt, durch die Verriegelung vorgenommen.

Die Marknagelung kann als minimalinvasive Osteosynthesemethode bezeichnet werden.

- Mit der Marknagelung findet im Regelfall eine natürliche sekundäre Kallusheilung statt.

- Die Marknagelung führt zu einer funktions- und zumindest teilbelastungsstabilen Osteosynthese.

\section{Klassische Marknagelung}

Von klassischer Marknagelung aus den 50er und 60er Jahren spricht man, wenn eine Marknagelung eine Schaftfraktur im mittleren Drittel stabilisiert. Vor der Marknagelung mit einem unverriegelten geschlitzten Rohrnagel wird so weit aufgebohrt, dass der enge klemmende Kontakt des Marknagels im Rohr des Röhrenknochens die Fraktur stabilisiert (Abb.3).

Die Indikation war auf das mittlere Schaftdrittel und quere oder kurze Schräg- und Spiralfrakturen beschränkt. Der endgültige Durchbruch und die Erweiterung der Marknagelung auf ein sehr breites Indikationsspektrum wurde durch die Verriegelung erreicht.

\section{Verriegelung}

Mit der Verriegelung wird ein Marknagel im körpernahen und körperfernen Fragment am Eintrittspunkt bzw. am Endpunkt zusätzlich quer oder schräg stabilisiert. Dies geschieht im Regelfall mit Schrauben oder schraubenähnlichen Bolzen.

Als weitere Elemente der Stabilisierung sind die diagonalen Schrauben, gewundene Klingen (Twisted Blade oder Spiralklinge) und andere Varianten möglich.

Die Verriegelungselemente werden durch im Nagel vorgegebene Löcher und Aussparungen eingebracht. Durch sinnreiche Techniken sind auch winkelstabile Blockierungen möglich (z.B. Spiralklinge des UFN, proximale diagonale Schraube des UTN).

Durch die Verriegelung von Marknägeln wird das Stabilisierungsprinzip wesentlich erweitert. Zusätzlich zur axialen kommt die Rotationsstabilität!

Bei der klassischen Marknagelung handelt es sich um einen offenen oder geschlitzten Rohrnagel, welcher sich im

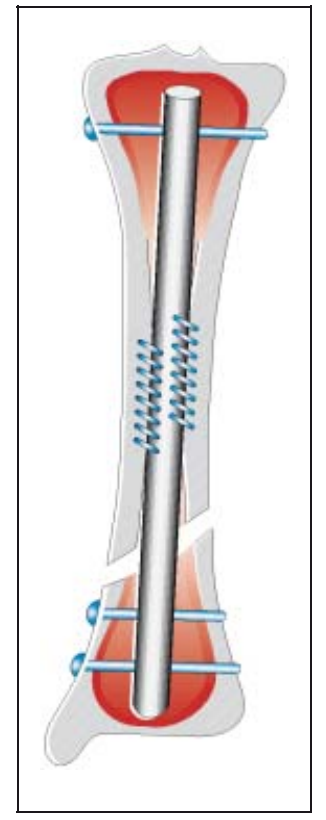

Abb. 5 Der aufgebohrte Abschnitt im Bereich des Schaftes (III) ist häufig mehrere Zentimeter von der Frakturzone entfernt. Somit muss vorsichtiges Aufbohren im Schaftbereich Nichtaufbohren in der Frakturzone bedeuten. Die Stabilität wird durch einen engen Kontakt im engen Schaftbereich erhöht (dargestellt am Beispiel der Tibia).

Markkanal verklemmt (deshalb das Wort Nagelung).

Die Verriegelungsnagelung ermöglicht eine Mehrpunktabstützung mit oder ohne innere Verklemmung (Abb.5).

Mit verriegelten Marknägeln können heute alle Frakturformen behandelt werden, welche zwischen den Verriegelungslöchern liegen oder im Einzelfall, z.B. beim distalen Femurnagel und beim proximalen Femurnagel hineinreichen.

\section{Proximale (insertionsnahe) Verriegelung}

Die proximale Verriegelung kann im Regelfall durch Zielbügel bewerkstelligt werden. Diese Zielbügel werden mit dem insertionsnahen Ende des Nagels verschraubt und können gleichzeitig als Einschlaginstrumentarium verwendet werden.

Anmerkung: Proximale und distale Verriegelung erhalten mit der Einführung des unaufgebohrten Humerusnagels (UHN), welcher vom Oberarmkopf und vom Ellenbogen her eingebracht werden kann und mit dem distalen Femurnagel, welcher vom Knie her eingebracht wird, eine neue Begriffsbestimmung, auf welche zu achten ist:

Bei diesen Nägeln, welche von körperfern eingebracht werden, ist die distale Verriegelung die insertionsnahe und die proximale Verriegelung die entfernt gelegene. Insofern sollte hier von ,insertionsnaher" und „insertionsferner" Verriegelung gesprochen werden.

\section{Distale (insertionsferne) Verriegelung}

Das insertionsferne Ende des Nagels verwindet und verbiegt sich bei langen Nägeln so, dass die für die Verriegelung vorgesehenen Löcher und Aussparungen nicht über einfache Bügelsysteme getroffen werden können. Bei kurzen Nägeln, wie z.B. den normal langen proximalen Femurnägeln (PFN) und bei kurzen distalen Femurnägeln (DFN), ist dies möglich.

Bei allen anderen Nägeln müssen für die distale Verriegelung sinnreiche Hilfsmittel (Ziel-Techniken) gefunden werden.

Wir unterscheiden heute vor allem:

- Verfahren auf röntgenoptischer Basis mit dem BV,

- Bügelsysteme, welche über Hilfsbohrungen an das Nagelende angepasst werden,

- in Zukunft rechnergestützte Navigation.

Die BV-gestützte Verriegelung arbeitet jeweils nach dem gleichen Prinzip, unabhängig, welches Bohrverfahren oder System verwendet wird.

Prinzipien der BV-gestützten distalen Verriegelung (Abb. 6):

Einstellen des zu treffenden Verriegelungsloches im Bildwandler orthograd, d.h. möglichst rund und im Zentrum des Bildwandlers (ansonsten Verzerrungsfehler).

- Einstellen des Monitorbildes seitengleich zum OP-Feld!

- Aufsetzen mit dem Bohrer und sonstigem Instrument auf den Eintrittspunkt.

- Durchbohren orthograd entlang dem "Zentralstrahl“ durch das vorgegebene Verriegelungsloch. Dies kann dann mehr oder weniger kontrolliert werden. Mit dem röntgendurchlässigen Winkelgetriebe der $\mathrm{AO} /$ Synthes funktionieren diese Schritte sehr einfach und wirkungsvoll.

\section{Aufgebohrte und unaufgebohrte Marknagelung}

Beide Methoden haben ihre Vor- und Nachteile sowie sich weit überlappende Indikationsspektren. Es handelt sich 

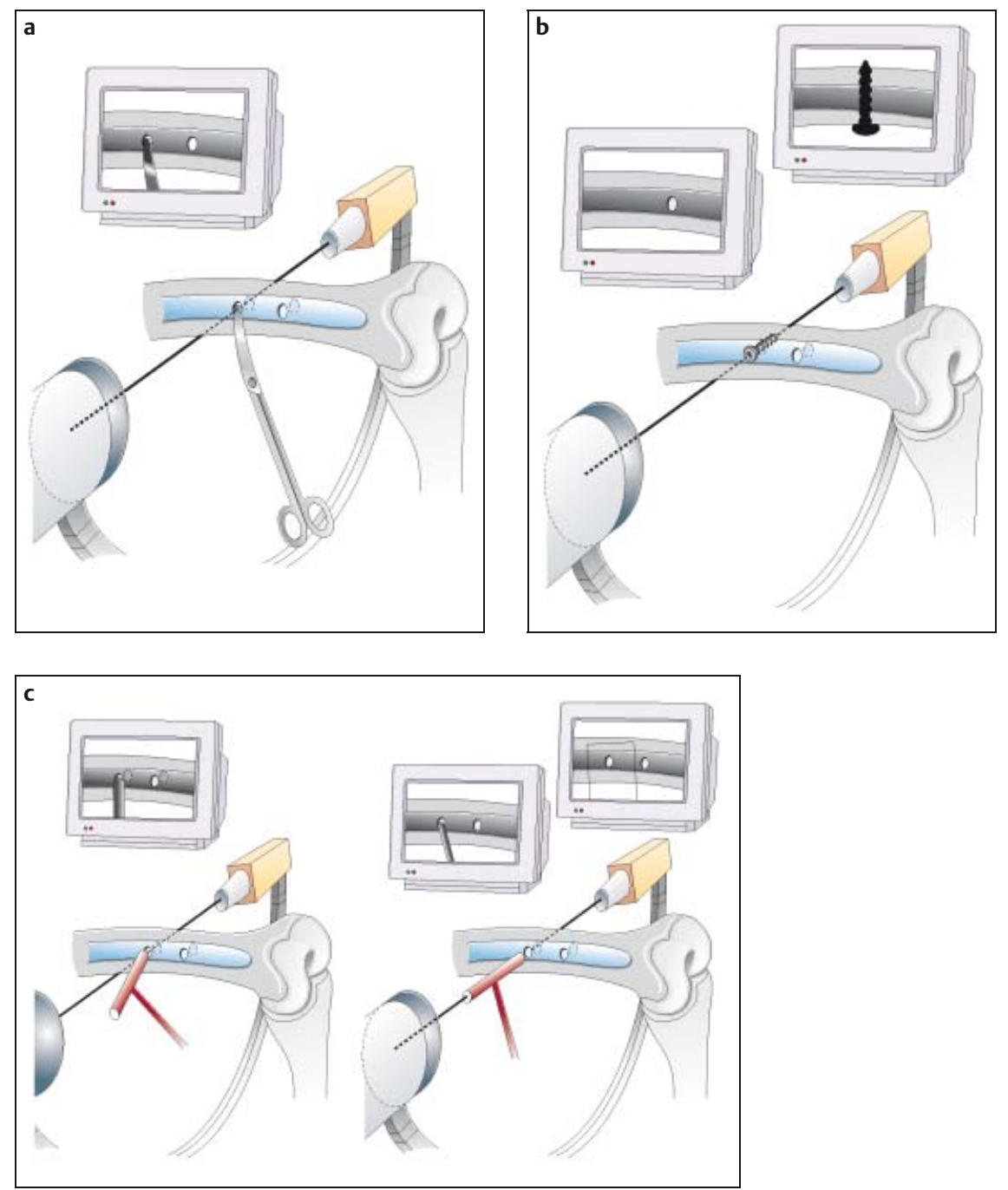

Abb.6a-c Grundprinzip der distalen Verriegelung mit dem röntgenoptischen Verfahren. (a) Darstellen des Verriegelungsloches rund im Bildwandler seitengleich mit dem Operationsfeld und Bestimmen des Eintrittspunktes. (b) Nach einer Stichinzision wird das Instrument in den „Zentralstrahl“ geschwenkt. Dieses Instrument kann eine Bohrbüchse, ein Bohrer in Freihandtechnik, ein röntgendurchlässiges Winkelgetriebe (von AO/ Synthes), ein Pfriem oder andere geeignete Instrumente sein. (c) Nach erfolgreicher Bohrung wird die Verriegelungsschraube oder der Verriegelungsbolzen eingebracht. Die muss in beiden Ebenen röntgenologisch kontrolliert werden.

aber nicht um ein Schwarz-Weiss-Bild, sondern um ein Bild mit Übergangszonen.

Aufgebohrt ist nicht gleich aufgebohrt, sondern es kommt darauf an, in welcher Art und wie intensiv das Aufbohren vorgenommen wird. Auf der anderen Seite sind bei der unaufgebohrten Marknagelung zum Erreichen einer ausreichenden Stabilität gewisse Regeln und Tricks einzuhalten.

Einigkeit besteht darüber, dass das Aufbohren eine Belastung des biologischen Systems „Markraum“ darstellt. Je intensiver und weniger schonend aufgebohrt wird, desto mehr wird die intramedulläre Blutzufuhr gestört und eventuell mehr oder weniger weitreichende Nekrosen im Bereich der Innenwand der Kortikalis gesetzt. Neben der Störung der Blutzufuhr wird durch das Aufbohren der Druckanstieg und ein venöses Emboliegeschehen gefürchtet. Dies ist vor allem am Femur ein Gesichtspunkt, welcher streng zu beachten ist. Durch viele Untersuchungen ist gezeigt worden, dass insbesondere mehrfachverletzte und polytraumatisierte Patienten sowie Patienten mit begleitendem Thoraxtrauma vor allem bei Femurfrakturen betroffen bzw. gefährdet sind. Insofern sind folgende Regeln einzuhalten:
Regeln beim Aufbohren für die Marknagelung:

Verwendung von scharfen Bohrern,

schrittweises Aufbohren mit $1 \frac{1}{2}$-mm-Abständen,

- Langsame Vorschubgeschwindigkeit mit eventuellen Pausen,

- nur so weit aufbohren wie notwendig,

- Zwischenfragmente und Segmentfragmente möglichst nicht bohrend überqueren.

Für die Beurteilung, wie schädlich das Aufbohren ist oder nicht ist, ist auch zu beachten, in welchem Bereich die Fraktur liegt und in welchem Verhältnis zur aufgebohrten Strecke. Bei den so häufigen distalen Femur- und Tibiafrakturen liegt der Bohrvorgang in Schaftmitte mehrere Zentimeter abgelegen vom „Epizentrum“ der Fraktur (Abb. 7).

Die unaufgebohrte Marknagelung leidet unter dem Nachteil einer möglichen Instabilität. Diesem kann durch folgende Regeln entgegengewirkt werden:

Regeln zur Erhöhung der Stabilität bei der unaufgebohrten Marknagelung:

- richtiges Kaliber verwenden,

- richtigen Eintrittspunkt wählen (besonders am Femur),

- die Länge proximal korrekt wählen,

- die Länge distal möglichst lang wählen (d. h. die distale Spongiosa als Lager und abstützenden Fußpunkt verwenden),

- gute und sorgfältig ausgeführte proximale und distale Verriegelung,

in der Nachbehandlung je nach Frakturform und verwendetem Implantat etwas mehr Vorsicht walten lassen als bei der aufgebohrten Marknagelung.

Beim Beachten dieser Regeln sollte es gelingen, die Aspekte der Biologie und Stabilität mit aufgebohrten und unaufgebohrten Nagelungen in Balance zu bringen.

\section{Solide Marknägel - geschlitzte Rohrnägel}

Die klassische Marknagelung wurde mit geschlitzten Rohrnägeln durchgeführt. Der Rohrnagel war geschlitzt, um die Verwindungs- und Torsionsstabilität zu erniedrigen, den Durchgang durch den Markraum zu verbessern und um durch eine leichte Kompression des Nagels einen stabilisierenden Klemmeffekt zu erzielen.

Solide Marknägel werden vor allem für die unaufgebohrte Technik verwendet, 
welche im Regelfall dünner sind. Durch die solide Ausführung werden gleiche Stabilitätswerte erreicht wie mit den dickeren geschlitzten Rohrnägeln. Da unaufgebohrte Marknagelungen nicht verklemmen sollen, konnte auf den Klemmeffekt verzichtet werden. Solide Marknägel haben den positiven Nebeneffekt, dass sie keinen Totraum haben. Dieser Totraum kann im Falle einer Infektion diese durch das Vorhandensein mindervaskularisierten Gewebes begünstigen. Durch die Rohrform stellt der Rohrnagel zusätzlich ein Drainagesystem von „oben bis unten“ dar. Es gibt erste Anzeichen, dass solide Marknägel für eine Infektion weniger anfällig sind und bei einer Infektion diese sich nicht im gesamten Markraum ausbreitet (diese Frage wird unabhängig von dem Merkmal aufgebohrt oder unaufgebohrt untersucht).

Regelhaft werden geschlitzte Marknägel für die aufgebohrte und solide Marknägel für die unaufgebohrte Technik verwendet.

Auch hier gibt es Ausnahmen von der Regel. So können (und wurden in der Anfangsphase) geschlitzte Rohrnägel in unaufgebohrter Technik eingeschoben. In Einzelfällen können solide Marknägel auch nach Aufbohren verwendet werden.

Für einzelne Spezialnägel wie z.B. den proximalen Femurmarknagel - welcher ein solider Nagel ist - oder auch bei sehr enger Markhöhle, kann es notwendig sein, aufzubohren. Daraus zeigt sich, dass die Einzelaspekte der Marknagelung nicht als Schwarz-Weiss-Bild zu sehen sind.

\section{Lagerung}

Die Lagerungen wurden in einer früheren Ausgabe des OP-Journals ausführlich beschrieben [3].

Die Lagerung ist abhängig von den Gewohnheiten der Schule und den Möglichkeiten (OP-Tisch/Extensionstisch) des jeweiligen Hauses.
Als Lagerung bei der Femurmarknagelung sind möglich:

- Extensionstisch in Rückenlage,

- Extensionstisch in Seitenlage,

- Normaltisch in Rückenlage,

- Normaltisch in Rückenlage mit abgespreiztem gesunden Bein.

\section{Proximale Femurmarknägel:}

- Extensionstisch in Rückenlage,

- Normaltisch.

\section{Distale Femurmarknägel:}

Normaltisch mit Möglichkeit der Beugung im Knie und Absenkung des Unterschenkels.

Für die Lagerung bei allen Marknagelungen ist ganz zu beachten:

Die Lagerung und Überwachung ist Aufgabe des Operateurs oder eines qualifizierten Vertreters.

- Es ist auf eine gute Abdeckung zu achten.

- Intraoperativ muss die Röntgenkontrolle im Bereich der proximalen Eintrittsstelle, der Fraktur und am körperfernen Ende möglich sein.

- Lagerung und Abdeckung sollten in einem Haus immer in gleicher Weise durchgeführt werden. Sie kann und sollte durch das Operationsteam, d. h. Operateure und OP-Pflegepersonal, geübt und festgelegt werden.

\section{Übersicht der Marknagelung langer} Röhrenknochen

Femur:

- Für die aufgebohrte Marknagelung geschlitzte Rohrnägel z.B. von der $\mathrm{AO} /$ Synthes Universalmarknägel.

- Für die unaufgebohrte Technik solide Marknägel (z.B. von AO/Synthes UFN) mit den verschiedenen proximalen Verriegelungen.

Proximaler Femur:

- Proximale Femurmarknägel mit dynamischer Hüftschraube wie Gammanagel, Classic Nail und von AO/Synthes PFN in normaler und langer Version.

\section{Distaler Femur:}

- Distale Femurmarknägel aller Hersteller.

- Von AO/Synthes mit der besonderen Verriegelungsmöglichkeit einer Spiralklinge.

\section{Literatur}

${ }^{1}$ Küntscher G. Praxis der Marknagelung. Schattauer, Stuttgart 1962

2 Müller ME, Allgöwer M, Schneider R, et al. Manual der Osteosynthese. 3. Aufl., Springer Verlag, Heidelberg 1990

3 OP-Journal, Sonderausgabe: OP-Tisch Lagerungen, Instrumenten-Tische. Thieme Verlag, Stuttgart 1995

Prof. Dr. med. D. Höntzsch

Leitender Arzt Abteilung Medizintechnische Entwicklungen

BG Unfallklinik Tübingen

Schnarrenbergstr. 95

72076 Tübingen

Prof. Dr. med. Dr. med. h.c mult. S. Weller

Engelfriedshalde 47

72676 Tübingen

Prof.-Dr. med. K. Weise

Ärztl. Direktor

BG-Unfallklinik Tübingen

Schnarrenbergstr. 95

72076 Tübingen 